\title{
Anfänge einer modernen China-Rezeption in deutschen Kulturzeitschriften um 1900
}

(vorgetragen in der Plenarsitzung am 9. Oktober 2009)

HeinRich Detering

Die folgenden Beobachtungen ergeben sich aus der Auswertung zunächst von „Westermanns Monatsheften“ (neun Beiträge) und der „Deutschen Rundschau“" (drei Beiträge) zwischen Januar 1899 und Februar 1901. Wegen der besonderen Relevanz für die Frage nach der Auseinandersetzung mit der Kultur Chinas wurde zusätzlich die von Maximilian Harden herausgegebene "Zukunft" für denselben Zeitraum ausgewertet, stichprobenhaft außerdem die populäre bürgerliche Unterhaltungszeitschrift „Die Woche“ herangezogen.

Wie nicht anders zu erwarten, stehen im Hintergrund dieser - keineswegs vorrangig politisch ausgerichteten - Beiträge der sogenannte „Boxeraufstand", seine Vorgeschichte und seine Folgen. Die Nachrichten vom Staatsstreich, von der gewaltsamen Rebellion und ihrer Niederschlagung 1899/1900 provozieren in den Kulturzeitschriften Fragen nicht nur nach den aktuellen Vorgängen, sondern vor allem nach deren Grundlagen und Voraussetzungen in der traditionellen chinesischen Kultur. Und erst sie haben diese Wirkung; denn auch wenn bereits die Besetzung der Kolonie Kiautschou 1898 ein merkliches China-Interesse in den Zeitschriften und auf dem Buchmarkt geweckt hat, so scheint es doch, als habe erst der gewaltsame Schock des Aufstands den deutschen Kulturzeitschriften die Wissensdefizite schlagartig zum Bewußtsein gebracht, die im deutschen Bildungsbürgertum im Hinblick auf China bestanden. Mehrere der hier ausgewerteten Beiträge thematisieren diesen Wandel des kulturellen Interesses selbst.

Im Juli 1900 wird der deutsche Gesandte von Ketteler durch antikolonialistische Aufständische in Peking ermordet; kurz zuvor, Anfang 1899, hat sich ein für die meisten europäischen Beobachter undurchschaubarer Staatsstreich ereignet, als dessen Drahtzieherin die in Europa überwie-

$\overline{1}$ Auf der Grundlage der von Susanne Friede und Christoph Jürgensen erschlossenen und ausgewerteten Bestände. 
gend zur unheimlich-exotischen Herrscherin dämonisierte Kaiserinmutter Tse-Si gilt. Kaiser Wilhelm hält vor Marinesoldaten in Wilhelmshaven jene von ihm selbst als markig und fulminant empfundene Ansprache, die als „Hunnenrede“traurige Berühmtheit erlangt hat. Gegen die Aufständischen soll in China eine exemplarische „Strafexpedition“ durchgeführt werden. In den deutschen Kulturzeitschriften führt das zu Diskussionen um die kulturelle Sendung des christlichen Westens in China, um Möglichkeiten und Grenzen einer politischen und ökonomischen Hegemonie und vor allem um die kulturellen Eigenarten und Traditionen dieses Landes selbst.

\section{Kultur und Kolonialismus: Meinungsverschiedenheiten in der „Deutschen Rundschau“"}

Bereits im Februar 1899, nach den ersten Nachrichten vom chinesischen Staatsstreich, gibt in der „Deutschen Rundschau“" Max (eigentlich Maximilian) von Brandt, der einstige deutsche Botschafter und zeitweilige Doyen des diplomatischen Corps in Peking, der schon seit den achtziger Jahren zum weithin angesehenen sinologischen Forscher und Autor geworden ist, einen zweiundzwanzig Seiten langen, kenntnisreichen Abriß der jüngsten chinesischen Geschichte. Der Text, der eine dreißigjährige Entwicklung umsichtig resümiert, mündet schließlich in die Ermahnung, ,sich in China nicht auf politische Experimente einzulassen“, sondern vielmehr auf „eine weitere Fortentwicklung ohne gewaltsame Umwälzungen "zu bauen (Band 98, S. 190).

Mit der Verschärfung der Krise verstummen solche Töne hier. In derselben Zeitschrift wird wenig später, im April 1900, von Wilhelm Grube unverändert wieder jene offensive Gangart befürwortet, die dem bewährten kolonialen Argumentationsmuster entspricht. Eine erfolgreiche „Culturvermittlung" bedeutet für ihn im wesentlichen eine Christianisierung der Chinesen, die allerdings - und allenfalls in dieser gönnerhaften Relativierung der alten Barbareiklischees werden dann doch Spuren der neuen, differenzierten Kenntnisse sichtbar - für eine solche Bekehrung auch ungewöhnlich günstige kulturelle Voraussetzungen mitbrächten. In der Quintessenz bleibt auch Grubes Perspektive dennoch ganz auf den unmittelbaren deutschen Eigennutz begrenzt. „Für uns Deutsche“, erklärt er,

[...] hat der Confucianismus heutzutage nicht nur rein theoretisches Interesse, vielmehr gewinnen, seit wir durch unsere Stellung in China in unmittelbare Beziehungen zu dem Chinesenthum getreten sind, die Kenntniß seiner Lebensanschauung und das Verständnis für seine geistige Eigenart eine eminente praktische Bedeutung. (Band 103, S. 96) 
Deutlicher gesagt: China „steht vor der Alternative, entweder der Cultur des Abendlandes gutwillig Zutritt zu gewähren [...] oder zu unterliegen" (S. 110).

Das ist während der Unruhen, aber noch unmittelbar vor dem Aufstand geschrieben. Schon sechs Monate später, nachdem die Schreckensnachrichten sich allgemein verbreitet haben, ändert sich die Linie des Blattes. Nun zwingen „die Ereignisse [...], mit welchen China die Welt überrascht und seit Monaten in peinlicher Spannung gehalten hat", mit einem Mal „unser deutsches Vaterland“, sich ernstlich mit dem zu befassen, was hier in der Überschrift die "Charakteristik des Chinesen“ genannt wird. Aus einem englischen Beitrag von Lady Blennerhassett ist der Beitrag übersetzt, der nicht nur neunzehn eng bedruckte Seiten umfaßt, sondern sich auch auf nicht weniger als vier gelehrte Werke (und weitere Aufsätze) bezieht, die zwischen 1898 und 1900 über chinesische Landeskunde, Kultur und Religion erschienen sind.

Gleichzeitig interessiert sich die „Deutsche Rundschau“ auch für die russischen, österreichischen und französischen Reaktionen auf das politische Drama; die „Wiener Zeitung“ wird ebenso referiert wie der Pariser „Temps“. (Und mit der Erwähnung von Paris kommt wiederholt auch die dortige Weltausstellung in den Blick, die nun nachdrücklich als „Friedensund Culturwerk" charakterisiert wird, so in der „Deutschen Rundschau“" im November und erneut im Dezember 1900.) Nach alldem fragt sie, tagespolitisch involviert, eindringlich und mit Nachdruck. Nur nach einem fragt sie eigentlich nicht: nach Kultur und Geschichte jenes fremden und anderen China, das mit den Unruhen auch für die deutschen Kolonialideologen unübersehbar geworden ist - bis im September 1900 erneut der besonnene Max von Brandt die Entwicklungen des Boxeraufstands zum Anlaß nimmt, ebendiese Position grundsätzlich in Frage zu stellen.

In seinem ganz auf die Tagesereignisse ausgerichteten Beitrag „Die chinesische Frage“ betont von Brandt die Notwendigkeit, ,sich über die Ziele der deutschen Politik klar zu werden“ und gegebenenfalls kluge „Beschränkung" zu üben (Band 104, S. 387). Und er fordert mit aller ihm zukommenden diplomatischen und gelehrten Autorität eine Selbstkritik des deutschen Kolonialismus genau dort, wo Wilhelm Grube sie im selben Magazin verweigert hat. Das geschieht gleich in den ersten Sätzen:

Noch sind die Augen der ganzen civilisirten Welt auf den ersten Act des Kampfes gerichtet, der im fernsten Osten zwischen der modernen Cultur der christlichen Völker und der uralten heidnischen Civilisation China's entbrannt ist, und schon zwingt uns ein furchtbares Ereigniß, an die eigene Brust zu greifen und uns zu fragen, wie weit 
wir berechtigt sind, uns als die Träger der wahren Bildung zu betrachten und in ihrem Namen das zu vernichten, was Jahrtausende geschont haben. (S. 384)

Schon die Wendung von "der uralten heidnischen Civilisation China's“ spricht Grubes kolonialistischer Argumentation - und der kaiserlichen Politik - die grundlegende Berechtigung ab. Nicht um den Gegensatz von Heiden- und Christentum geht es bei ihm und schon gar nicht um deren Gleichsetzung mit Barbarei und Kultur, sondern um christliche und „heidnische Civilisation“. Die Unterstellung einer spezifisch chinesischen „Bildung" und die Betonung ihres hohen Alters verbinden die Forderung nach politischer Selbstkritik sogleich mit derjenigen nach einer Überwindung der, was diese ferne Zivilisation angeht, eigenen Unbildung.

\section{2. „Unter dem Drang der Ereignisse“: „Westermanns Monatshefte“ und ihr Herausgeber}

Auch in „Westermanns Monatsheften“ löst die politische Katastrophe ein nicht nur neues, sondern (auch in der Selbstwahrnehmung) durchaus neuartiges Interesse aus. Hier wird über die Tagesaktualitäten hinaus zurückgefragt nach kulturellen Grundlagen. Zwar ist das Interesse einer gebildeten deutschen Öffentlichkeit an chinesischer Kultur seit Schillers, Goethes und Humboldts Hinwendung zur chinesischen Kultur nie ganz abgerissen. Von Schillers vermeintlichen Chinoiserien in "Turandot" (die der chinesische Germanist Yuan Tan jüngst als untergründige Auseinandersetzung auch mit dem genuin Fremden der anderen Kultur interpretiert hat) ${ }^{2}$ über Goethes „Chinesisch-deutsche Jahres- und Tageszeiten“ (die ihrerseits weit über die exotische Maskerade des Weimarer Mandarins hinaus durchgängig, und in einem bis heute zumeist erheblich unterschätzten Umfang, mit „chinesischen “ Kulturformen spielen) ${ }^{3}$ bis zu dem bereits im Laufe des 19. Jahrhunderts über Frankreich vermittelten Interesse an Konfuzianismus und Taoismus gehören literarisch und philosophisch-religionsgeschichtlich ausgerichtete Texte jedenfalls am Rande auch zum deutschen Bildungskanon des ausgehenden 19. Jahrhunderts. Um die Jahrhundertwende gewinnt dieses Interesse, so läßt sich der dominierende Eindruck der untersuchten Zeitschriftenbeiträge resümieren, schlagartig eine ganz neue Dringlichkeit.

2 Dazu Yuan Tan. Dr. Tan ist für seine Hilfe zu danken. Zum weiteren ideologischen Kontext der kolonialen und der postkolonialen literarischen China-Rezeption im wilhelminischen Deutschland vgl. auch die demnächst in der Göttinger Reihe „Palaestra“ erscheinende Studie von Wolfgang Struck.

3 Dazu noch immer grundlegend der Aufsatz von Debon. 
Nun wird die Frage nach der chinesischen Kultur von einem akademischen oder ästhetischen Sonderinteresse zu einer politischen Frage von politischer Bedeutung. Sie richtet sich zunehmend auf die literarischen und künstlerischen Zeugnisse einer longue durée chinesischer Mentalität, Denkweisen, kultureller Prägungen. ${ }^{4}$ Dabei wird die Kluft zwischen den drängenden politischen Tagesfragen und einem als mangelhaft empfundenen Grundwissen über chinesische Traditionen wiederholt zum Thema gemacht.

Noch kurz vor den dramatischen Ereignissen in China, im Januar 1899, wendet sich der als einschlägig publizierender Beiträger den Lesern von „Westermanns Monatsheften“ vertraute Max von Brandt mit dem Aufsatz „Ein Kapitel aus der chinesischen Kunstgeschichte. Symbolik und Bilderschrift", ganz ohne politische Untertöne, an ein kunstliebendes und wohlhabendes Publikum (Band 85). Auf siebzehn großformatigen und reich illustrierten Seiten versucht er, eine kunstgeschichtliche Einführung in die Symbolsprache chinesischer Porzellanmalerei, Stickerei und anderer Gebrauchskunst zu geben - zunächst für Museumsbesucher, die vor den exotischen Exponaten weitgehend verständnislos stehen, dann aber auch für Kunden der Kunsthandlungen, die dank der Kolonien nun zunehmend Chinesisches einführen und feilbieten. Die damit zunächst durchaus zweckgerichtete Abhandlung führt dabei immerhin in Grundzüge der chinesischen Kosmologie, Astrologie und in diesen Grenzen auch Philosophie ein. Das kulinarische Interesse an dekorativen Aspekten der Chinoiserie provoziert Fragen nach den Eigenarten der als so dekorativ empfundenen Kultur selbst, nach ihren Voraussetzungen und Erscheinungsformen.

Es ist aufschlußreich zu sehen, wie sich zwei Jahre später am selben Ort, in einer mehr als zwanzig Seiten umfassenden Abhandlung über Peking einst und jetzt, von Brandts Connaisseurton und seine durchaus selbstsichere koloniale Perspektive auf die chinesische Kultur gewandelt haben. Nun, im Februar 1901, fragt er in „Westermanns Monatsheften“"vor allem nach dem gegenwärtigen Wandel in China. Genauer: Er fragt nach dem, was er mit spürbarer Erregung und mit offenem Bedauern „die Dimensionen einer niedergehenden Kultur" nennt, den Gegensatz zwischen „einer mächtigen Vergangenheit und einer verlotterten Gegenwart" (Band 89, S. 650).

Wie die Berichte, so die Rezensionen. Hier ist für alle Chinathemen in „Westermanns Monatsheften“ der Herausgeber persönlich zuständig, Friedrich Düsel. Sieben Rezensionen zu Büchern über Themen, die (auch)

4 Stichproben in der weniger anspruchsvollen, sehr populären und gewissermaßen zwischen Kulturzeitschrift und yellow press changierenden Zeitschrift „Die Woche“ bestätigen die hier sichtbar werdende Tendenz. Auch dort gewinnen in dieser Zeit chinesische Gartenkunst, Architektur und Alltagskultur ein neues, wenngleich hier sehr viel oberflächlicher artikuliertes Interesse. 
mit China zu tun haben, veröffentlicht er in seinem Magazin zwischen Januar 1899 und Februar 1901.

Nachdem Düsel schon im November 1898 von „Werken über das Gebiet der deutschen Niederlassung in China" berichtet hat, beschäftigt er sich im Januar 1899 mit Ernst von Hesse-Warteggs auf einer 1897/98 unternommenen Reise beruhenden Berichten über die kolonialen Stützpunkte, die der Rezensierte einigermaßen hochgreifend „Deutsch-China“ nennt. Der Reisende führe seine Leser, so lobt der Rezensent, bis hinein „ins heilige Land von China und vom Jangtsekiang nach Peking“ mitsamt „den Geburts- und Grabstätten des Religionsstifters Confucius und seiner Apostel“ und lasse dabei „kein Gebiet des öffentlichen und privaten Lebens unberücksichtigt“. Da er sich „mit besonderer Andacht in das intime Alltagsleben des chinesischen Haushaltes“ versenke, mache er „das chinesische Milieu mehr noch von innen als von außen lebendig". Im Mittelpunkt der kurzen Besprechung aber steht ein anderer Gedanke: Hesse-Wartegg habe unterwegs auch

alle Orte besucht, die für Deutschland von irgend welchem Interesse sein konnten: die großen Städte und Warenmärkte, die Kohlen- und Industriegebiete, die Sitze der deutschen Mission in Schantung, sowie die Gegenden, durch die die geplanten deutschen Eisenbahnen führen werden. (Band 85, S. 542)

Im Oktober desselben Jahres wendet sich Düsel in einer Sammelrezension zu neuen Reisebüchern demselben Thema zu. Denn: „Es wäre ein Wunder, wenn heute in einer noch so kurzen Übersicht über Reiselitteratur China nicht vertreten wäre". Mit Nachdruck stellt er dem von Ernst Ruhstrat veröffentlichten Band „Aus dem Lande der Mitte“, „der wesentlich praktische Zwecke der Belehrung verfolgt und in der That schnell über die wichtigsten Zustände und Einrichtungen Chinas unterrichtet", Max von Brandts umfangreiche Abhandlung „Die chinesische Philosophie und der Staatsconfucianismus“ gegenüber, in der die „Entwicklungsgeschichte der verzwickten chinesischen Religionslehren des Confucius, des Mencius und des Laotze sowie der daraus abgeleiteten staatlichen Moralphilosophie“ umfassend und gründlich dargestellt werde. Der Vergleich geht zum Nachteil Ruhstrats aus: Man empfindet, bemerkt Düsel, „allzu schmerzlich den gewaltigen Unterschied zwischen einer gutgemeinten Dilettanten- und einer gediegenen, wertvollen Gelehrtenarbeit". Und so konzentriert er sich auf ein Referat der genannten religiösen Lehren in ihren Grundzügen und auf die von Alfred Forke herausgegebene Anthologie „Blüten chinesischer Dichtung “, in der Tat einen frühen Klassiker der deutschen Rezeption chinesischer Lyrik. Dabei bemüht er sich, den Begriff des „Tao“ mit dem 
gebildeten deutschen Lesern vertrauteren des „Logos“ zu erklären, erläutert die Bedeutung der konfuzianischen Philosophie für die ,jetzt regierende mandschurische Herrschaft" und versucht die „Individualität" des überragenden Dichters Li-Tai-Po behelfsweise durch einen Vergleich mit dem deutschen Lesebuch zu erklären: „Seine Treuherzigkeit erinnert manchmal an Uhland“ - was mit der Charakterisierung "der geniale Vagant" nicht leicht zusammenzubringen ist. Vor diesen ernsthaft auf eine Annäherung an chinesische Traditionen jenseits der kolonialen Tagesinteressen zielenden Abschnitten allerdings hat Düsel noch Ruhstrats praktischem Handbuch die offenbar keineswegs maliziös gemeinte Bemerkung mitgegeben:

Besonders für Marineoffiziere wird das Buch [Ruhstrats] zu empfehlen sein - freilich nur wenn sie verstehen, manches, was der Verfasser nach englischen Quellen wiedergegeben hat, sich selbst unter deutsche Gesichtspunkte zu rücken. (Band 87, S. 140 f.)

Und auch Düsels knappe Rezension der kulturpsychologischen Studie „Chinesische Charakterzüge" von Arthur H. Smith im Dezember 1899 betont ausdrücklich die deutsche Perspektive bzw. ökonomische Tagesinteressen, genauer: den Nutzen des „unter Gesichtspunkten des praktischen Lebens zusammengestellt[en]" Bandes für den deutschen Kaufmann. (Band 87, S. 439)

Die Spannung von aktuellem Kolonial- und weiter ausgreifendem Kulturinteresse tritt noch deutlicher hervor, wenn derselbe Rezensent ein knappes Jahr später, also noch ganz unter dem Eindruck des „Boxeraufstands“ und seiner Niederschlagung, am selben Ort eine von Alfred Grünwedel verfaßte Darstellung der „Mythologie des Buddhismus“ vorstellt und dabei zumindest den Eindruck erweckt, als halte er auch das gegenwärtige China für ein buddhistisches Land. Düsel holt zunächst weit aus:

Der Strom unserer [der Deutschen] großen geschichtlichen Interessen geht heute anders als dereinst im Mittelalter. An die Stelle Italiens, das einstmals unsere politische und künstlerische Phantasie in so hohem Grade beschäftigte, drohen ferner liegende, in unseren Augen barbarischer geartete Länder zu treten und - freilich in ganz anderem Sinne! - für lange unsere gespannte Aufmerksamkeit und Wachsamkeit in Anspruch zu nehmen. Es sind das die Länder des Buddhismus: vor allem Japan und China. Da wird denn ein reich illustriertes Werk willkommen geheißen werden, das die Mythologie des Buddhismus darstellt [...]. (Band 89, S. 143)

Schon im Dezember ist „Westermanns Monatsheften “ das Erscheinen einer erweiterten zweiten Auflage von Ernst von Hesse-Warteggs schon 1896 besprochenem Reisebuch "China und Japan" eine erneute Rezension wert. Denn nun habe der Verfasser auch die mittlerweile ihrerseits in Buchform 
geschilderten „,neue[n] Beobachtungen und Eindrücke [...] aus Schantung und ,Deutsch-China' [...] hier für die allgemeine Charakteristik geschickt verwertet". Diese aktuellen Kenntnisse seien, so schreibt Düsel nun, dringend an der Zeit. „Wie gerufen“, komme „gerade jetzt“ diese Neuauflage, denn: „Über die Aktualität des Werkes braucht heute kein Wort verloren zu werden". Gerade die aktuellen Ereignisse aber erzwingen nun eine Auseinandersetzung mit den nicht unmittelbar aktuellen Traditionen Chinas. Deshalb fügt Düsel seiner Empfehlung von Hesse-Warteggs Buch den Hinweis auf ein Werk hinzu, das sehr viel weiter in jene Bereiche ausgreift, in die Düsel selbst sich schon in seinen Empfehlungen Brandts und Forkes bewegt hat - und unterstellt dessen Verfasser eben dieselbe Wahrnehmung eines verbreiteten und nun eilig aufzufüllenden Kenntnisdefizits:

Nicht mehr und nicht weniger als eine vollständige Encyclopädie der Religion und Kultur Chinas sucht ein starker Band zu geben, den Ferdinand Heigl soeben [...] hat erscheinen lassen. Das Werk hat unter dem Drang der Ereignisse augenscheinlich etwas eilig abgeschlossen werden müssen [...]. (Band 89, S. 451)

Wie dringlich „unter dem Drang der Ereignisse“ der aktuelle Informationsbedarf nach kulturhistorischer Vertiefung verlangt, demonstriert Düsel erneut gleich im folgenden Monat. Die erweiterte Neuauflage eines zweibändigen Reiseberichts, den der Korrespondent der „Frankfurter Zeitung“ unter dem Titel „Ein Sommer in China“ vorlegt, hat sich gegenüber der Erstausgabe entscheidend gewandelt. Hat diese den Rezensenten mit ihren während einer Reise 1898 gewonnenen „scharfe[n] Beobachtungen, insbesondere über die wirtschaftlichen Verhältnisse “ überzeugt, so berücksichtigt die Neufassung die aktuellen Ereignisse. Daß sie damit „allen denen besonders willkommen sein wird, die über das Reich der Mitte und seine rätselhaften Kulturzustände in einer leichten feuilletonistischen Form unterrichtet sein möchten, ohne doch der Gefahr ausgesetzt zu sein, allzu flüchtige Augenblicksskizzen zu erhalten": das ist auch dadurch gewährleistet, daß der Autor (wie Düsel in ihrerseits bezeichnender Selbstverständlichkeit vermerkt) zusammen mit der Darstellung der unmittelbaren Gegenwart einen Einblick in die klassische chinesische Literatur gibt:

Die zweite Auflage berücksichtigt schon die Neugestaltung der Verhältnisse nach dem Boxer-Aufstande und fügt ein sehr interessantes Kapitel über chinesische Dichter hinzu, von denen charakteristische Proben in Übersetzungen mitgetheilt werden. (Bd. 89, S. 590. Meine Hervorhebung).

Nirgends wird die Wandlung der Interessen in diesen Monaten pointierter und beiläufiger sichtbar als in diesem „und“. Wo eben noch die „wirtschaftlichen Verhältnisse“ im Zentrum standen, da muss jetzt, unter dem erschüt- 
ternden Eindruck der „Neugestaltung der Verhältnisse“, nach Kunst und Literatur gefragt werden. So überrascht es nicht, daß Düsel dem Lob dieses Bandes sogleich die Empfehlung eines weiteren Werks Max von Brandts folgen läßt, der gerade als Diplomat in Peking zum kulturgeschichtlich gelehrten China-Kenner geworden ist. Nun wird er zum Vor- und Leitbild einer über den Tag hinaus denkenden und eben deshalb für den Tag nutzbringenden Auseinandersetzung mit China:

Zweifellos das Hervorragendste, weil Selbständigste seinem litterarischen und politischen Charakter nach, was uns dieses Jahr über Ostasien beschert hat, sind die Erinnerungen, die M[ax] v. Brandt unter dem Titel Dreiunddreißig Jahre in Ostasien [... ] soeben erscheinen läßt. Der Verfasser, der allbekannte frühere Vertreter Deutschlands in Peking, bedarf einer Empfehlung kaum, zumal in dieser Zeitschrift nicht, die in der glücklichen Lage war, ihren Lesern im Laufe der letzten Jahre mehr als einen seiner sachkundigen, tief eindringenden Artikel über China und chinesische Zustände zu vermitteln. [...] Wer die historischen Voraussetzungen kennen lernen will, aus denen sich die heutigen Zustände entwickelt haben und sich erklären lassen, der wird dieses Werk nicht umgehen können. (Ebd.)

Wiederum einen Monat später, in seiner letzten in unserem Untersuchungszeitraum erschienenen Rezension in „Westermanns Monatsheften“, geht Düsel - unter ausdrücklicher Berufung auf seine vorangegangene Rezension von Brandts „Dreiunddreißig Jahre“ - auch über diese Perspektivenerweiterung noch einmal energisch hinaus. In einer nun erstmals ausschließlich Neuerscheinungen über China gewidmeten Sammelrezension (in den bisher referierten Rezensionen ist China noch immer nur ein Thema unter anderen gewesen) kritisiert er im Februar 1901 die Publikationslage:

Die jüngste Litteratur über China, das plötzlich durch eine fanatische Bewegung, deren Folgen noch gar nicht abzusehen, die Blicke der Welt auf sich gezogen hat, läßt an Reichhaltigkeit und Gediegenheit vieles zu wünschen übrig. Es soll damit kein Vorwurf ausgesprochen sein; denn die wirklichen, zuverlässigen Kenner des Landes - soweit bei den rätselhaften Verhältnissen von „Kenner“ überhaupt die Rede sein kann - haben meistens bereits vor drei Jahren, bei der bedeutsamen Besetzung Kiautschous, das Wort ergriffen $[\ldots]$.

Und dann erinnert Düsel unter anderem an die Publikationen von Ernst von Hesse-Wartegg und Max von Brandt. Die in den aktuellen Umbrüchen sich abzeichnende Erkenntnis aber, daß womöglich selbst diese bislang als Autoritäten unangefochtenen „Kenner" von den jetzt sichtbar aggressiv werdenden „rätselhaften Verhältnissen“ noch viel zu wenig wissen, setzt jetzt ganz neue Wissensstandards. So gern Düsel deshalb „den frischen Gegenwartsstandpunkt" von Ernst Schotts Büchlein „Die Wirren in China und ihre Ursachen“ empfiehlt, das „namentlich über die Boxer und die abendländi- 
schen Beziehungen Chinas manches wissenswerte" präsentiere, so informativ er eine anonym erscheinende Heftreihe über aktuelle, „militärisch und politisch" relevante Zusammenhänge findet, so notwendig erscheint ihm doch eine zumindest etwas weiter in die Vorgeschichte ausgreifende Darstellung der "Taiping-Revolution in China“, die in "scheinbar nur losem Zusammenhang mit den augenblicklichen chinesischen Ereignissen steht", aber gerade deshalb „manche wertvolle Lehre und Erkenntnis auch für die gegenwärtige Lage" eröffnet (Band 89, S. 731 f.).

Bei alldem bleibt der kolonialistische Standpunkt, von dem aus Düsel seine Besorgnisse und Forderungen formuliert, gänzlich unerschüttert. Die vertieften kulturgeschichtlichen Kenntnisse, die er von Wissenschaft und Publizistik fordert und mit seinen Ausführungen über chinesische Religion, Philosophie und Literatur zumindest ansatzweise selbst zu vermitteln sucht, werden entschieden instrumentell verstanden: Nur wer wirkliche Kenntnisse über China besitzt, kann den bedrohlichen Aufständen wirksam begegnen. Mit geradezu programmatischer Deutlichkeit wird das am Ende dieser Übersicht über „Die jüngste Litteratur über China“ formuliert.

Da geht es um Eugen Wolfs unterhaltsames Reisebuch „Meine Wanderungen [... ], deren erster Teil sich Im Inneren Chinas bewegt". So lobenswert auch hier der „frische Darstellungston“ und „die leichte Plauderkunst“ des Verfassers erscheinen - im Kern dieser Buchempfehlung geht es um die kolonialpolitische Grundhaltung, die den Anlaß zu Wolfs Reisen gegeben hat und die allein auch für den Rezensenten von „Westermanns Monatsheften" alles Interesse an China legitimiert:

Der Verfasser gehört zu denen, die als erste die dringende Notwendigkeit eines baldigen Stützpunktes für unseren Handel im Osten Asiens bereits im Frühjahr 1896 erkannt und maßgebenden Kreisen darüber Vortrag gehalten haben. [.. . ] Er will in erster Linie bei unserer Jugend das Interesse für außereuropäische Länder stärken und dadurch dazu beitragen, uns in den Stand zu setzen, „nicht nur Berater der Völker über den ganzen Erdball, nicht nur die Berufensten in der hohen Politik zu sein und zu bleiben, sondern auch im Welthandel die allererste Stelle zu erreichen und zu halten." Der Entwicklung unseres überseeischen Handels und unserer Industrie wendet daher das Wolfsche Buch in allererster Linie sein Interesse zu [...].

Und das heißt für den Rezensenten, mit einem unerwartet pathetischen Resümee: „der Liebe für das ,größere Deutschland', das heute der vornehmste Zielpunkt unserer weltpolitischen Ideale ist“ (ebd.).

Die aktuelle Bedrohung ebendieser kolonialen, ökonomischen wie kulturellen Hegemonialansprüche durch die chinesischen Unruhen hat also bei diesem Rezensenten in "Westermanns Monatsheften“ ein nachdrücklich auf die longue durée der chinesischen Kulturgeschichte gerichtetes Interesse 
provoziert - das sich aber nur, mit eher sich steigerndem Nachdruck als selbstkritischer Reflexion, in den Dienst einer verbesserten Kolonialpolitik stellen soll.

Derselbe Interessenwandel aber kann um dieselbe Zeit auch eine dieser Ideologie genau entgegengesetzte Tendenz hervorbringen; die neue kulturgeschichtliche Neugier kann sich gegen die ideologischen Prämissen der Kolonialpolitik selbst wenden. Das geschieht dort, wo die in der „Deutschen Rundschau"von Wilhelm Grube noch mit merklicher Mühe unterdrückte Frage kolonialismuskritisch neu akzentuiert wird: die Frage danach, warum eine religiös, philosophisch und künstlerisch so traditionsreiche und hochentwickelte Kultur überhaupt einer kolonialen Domestizierung bedürfe. In Maximilian Hardens - freilich von vornherein kaiserkritischer - „Zukunft“ wird sie am nachdrücklichsten gestellt.

\section{Kultur versus Kolonialismus: Maximilian Hardens „Zukunft“}

Hardens bei durchaus konservativer Grundhaltung notorisch kaiserfeindliche und im politischen wie im literarischen Feld gleichermaßen wirkungsreiche Zeitschrift - die etwa für den am Grenzbereich von Literatur und Journalismus lebhaft interessierten jungen Thomas Mann ein erklärtes Vorund Leitbild war - hat schon im Februar 1900 spöttisch auf die deutsche Erregung um den chinesischen Staatsstreich des Vorjahres reagiert. Auch Harden veröffentlicht da einen Essay unter der Überschrift „Tse-Si“. Doch er schlägt ganz andere Töne an als sowohl die „Deutsche Rundschau“ als auch „Westermanns Monatshefte“:

Von Zeit zu Zeit spukt jetzt ein orientalisches Gespenst durch die Spalten unserer geliebten Zeitungen, dann taucht irgend eine abenteuerlich klingende Kunde von blutigen Gräuelthaten der das Reich der Erdmitte regirenden Dame auf. (Band 30, S. 321)

Harden nimmt die spöttisch notierten exotischen Ängste zum Anlaß für eine grundsätzliche Forderung, die er auch in den folgenden Monaten programmatisch vertreten wird:

Nun sind, seit in Schantung die deutsche Flagge weht, die chinesischen Zustände für uns doch einigermaßen wichtig geworden; und wenn deutsche Inseratenfarmer sich schon nicht entschließen können, ernsthafte Berichterstatter nach Ostasien zu schicken [...], dann sollten sie wenigstens darauf halten, daß die in der berliner Meinungsfabrik [...] Bediensteten die [...] während der letzten Jahre über China veröffentlichten Bücher lesen und sich das von Landeskundigen gefundene Material aneignen. Wäre diese geringe Mühe schon früher aufgewandt worden, dann hätte man den Buddhismus nicht für die chinesische Staatsreligion [... ] gehalten. (S. 321 f.) 
Dieselbe Forderung wurde auch von Friedrich Düsel in „Westermanns Monatsheften " immer wieder formuliert - er selbst war dann ja allerdings auch einer derjenigen, die den Buddhismus für die chinesische Staatsreligion zu halten schienen. Mit ähnlich demonstrativer Neugier und Anteilnahme referiert daraufhin auch Harden, wie nebenan Düsel, nun aus „Büchern und Aufsätzen landeskundiger Leute“, auf der Suche nach einem älteren und weiteren China als dem Land zwischen Schantung und Kiautschou.

Mit dem kaiserlichen Säbelrasseln im Juli gewinnen diese zunächst noch vergleichsweise allgemein formulierten Maximen eine kolonialismuskritische Aktualität und Relevanz. „Die deutsche Kriegsflagge weht auf dem Weltmeer" (Band 32, S. 49), beginnt Hardens Artikel im Juli 1900 - und dann wird ausgiebig aus der kaiserlichen Rede und den folgenden Stellungnahmen des Hofes zitiert. Präzise, bitter, zuweilen maliziös (und so politisch überaus detailliert) hält Harden dem deutschen Herrscher die Widersprüche einer Argumentation vor, wie sie auch der kaisertreue Wilhelm Grube in der „Deutschen Rundschau“ favorisiert hat. „Der Kaiser“, so höhnt Harden, „will einen Rachekrieg führen und zugleich die Asiaten die milde Wunderkraft des Christenkreuzes lehren“ (S. 51); er "will Weltpolitik größten Stils betreiben“ und den „deutschen Besitz in Ostasien beträchtlich mehren" (S. 52) - und doch besitzt er von der kulturellen Eigenart des fernen Landes nicht einmal eine vage Vorstellung.

Damit kommt nun wieder die Argumentationsfigur ins Spiel, die im Nachholen grundlegender Kulturkenntnisse die wesentliche Voraussetzung einer notwendigen neuen Chinapolitik sieht. Nur scheint diese neue Politik nun eine grundlegende Abkehr vom Kolonialismus selbst einzuschließen. Harden:

Heute noch, wie vor Humboldts Tagen, ist China den Deutschen ein unbekanntes Land. Mancher Gelehrte hat in der Sammlung der Sacred Books of the East den TaoTe-King gelesen, Lao-Tses ehrwürdige Chinesenbibel, und mit heißem Bemühen die confucianische Sittenlehre studiert [...]. (S. 53)

Das „Wesen des gelben Volkes“ aber ist dabei „auch gebildeten Deutschen verborgen“"geblieben - „und so konnte der Glaube aufkommen, die Chinesen seien Barbaren, denen mit Pulver und Blei die Grundbegriffe civilisierter Menschheit beigebracht werden müssten“ (ebd.).

Noch im selben Monat zieht Harden aus dieser Einsicht eine bemerkenswerte literarische Konsequenz. Am 21. Juli veröffentlicht er unter der Goetheschen Überschrift „Chinesisch-Deutsche Jahreszeiten“ eine siebenseitige Zitatcollage aus Texten der Bibel, Lao-Tses, Nietzsches, Goethes, 
Schopenhauers, Montesquieus, Renans und anderer, die alle entweder von China oder von den Prinzipien toleranter Politik handeln. Wie die Überschrift nicht nur an eine lange Tradition deutschen Chinainteresses erinnert, sondern überdies auch der kaiserlichen Brutalität die Offenheit der Weimarer „Weltliteratur-“ und Humanitäts-Leitbilder entgegensetzt, so macht diese Blütenlese aus dem jäh aufgebrochenen Gegensatz von kulturellem und kolonialem Chinainteresse geradezu ein politisches Programm.

Gegen die staatsoffizielle Kolonialideologie läßt Harden so ein polemisches und programmatisches Gespräch über die Kontinente hinweg entstehen. „Das Volk wird unruhig, weil die Großen sich unsinnig gebärden“ (Band 32, S. 100), läßt er Lao-Tse sagen (der auf dem Weg über die französische Rezeption des 19. Jahrhunderts eben erst im Begriff ist, zum Modephilosophen der deutschen Frühmoderne zu werden; vgl. Weber, S. 27). Und den liberalen französischen Theologen, Religionswissenschaftler und Schriftsteller Ernest Renan läßt er über die Jahrhunderte hinweg antworten, die Trennung der Menschheit in Rassen sei „nicht nur wissenschaftlich unhaltbar“, sondern müsse früher oder später „zu Vernichtungskriegen führen, zu zoologischen Kriegen, wie wir sie aus dem Reich der Nager und Fleischfresser kennen." (S. 101 f.)

Zwei Ausgaben später folgt eine ähnliche Collage von Berichten und Anekdoten über Kaiser der chinesischen Geschichte, über die philosophischen Weisheiten des Mentius und des Konfuzius und so fort. Am Ende dieser Reihe steht die Geschichte von jenem Kaiser Tsi-Schi-Hoang-Ti, der „die Bücher verbrennen und vierhundert nörgelnde Literaten lebendig begraben“ ließ: „Seine Regirung aber", so lehrt das chinesische Exempel die deutschen Leser, „gilt als eine der unheilvollsten, die das Reich je kannte“ (Band 32, S. 224).

Die damit zu konstatierende Wendung der in zunächst durchaus instrumenteller Absicht aus dem politischen Kolonialkonflikt erwachsenen kulturellen Neugier gegen ebendiese Kolonialpolitik zeichnet sich hier nur in den wenigen für unsere Untersuchung umfassend ausgewerteten Kulturzeitschriften ab. Diese gehören jedoch, auch das ist festzuhalten, zu den verbreitetsten und intellektuell einflußreichsten Periodika der Epoche.

\section{Die Ursprünge des Wang-lun: Literarische Folgen}

Um genau dieselbe Zeit, in der in den deutschen Kulturzeitschriften diese Auseinandersetzungen geführt werden, geht der deutsche Wissenschaftler Richard Wilhelm in die deutsche Kolonie Tsingtau. Dieser angesehenste 
deutsche Sinologe seiner Zeit ist nach einem Studium in Tübingen und einer lebensbestimmenden Freundschaft mit dem späteren religiösen Sozialisten Christoph Friedrich Blumhardt 1899 als Pfarrer und Missionar in die deutsche Kolonie in Tsingtau gegangen; 1924 kehrt er dann als Gelehrter nach Deutschland zurück. Mit seinen in Tsingtao erarbeiteten Übersetzungen von Grundtexten der klassischen chinesischen Philosophie gerät er in der deutschen Rezeption unversehens auf jene interkulturelle Bühne, die hier von Maximilian Harden und anderen gezimmert worden ist. Dieser deutsche Theologe, der zur Jahrhundertwende als evangelischer Missionar in die Kolonie Tsingtau gegangen war, hat später erklärt, er sei dort nicht zum Verkünder des Christentums unter den Chinesen geworden, sondern von Tsingtau aus zum wirkungsvollsten Verkünder chinesischer Weisheit unter den Deutschen. Seine Übersetzungen beziehen ihr enormes Wirkungspotential daraus, daß sie das missionarische Prinzip der Inkulturation umkehren, indem sie nicht mehr die christliche Heilsbotschaft in Sprachbildern der chinesischen Tradition ausdrücken, sondern die chinesische Tradition durch die Verbindung mit christlichen Sprachbildern innerhalb einer christlich bestimmten Kultur verstehbar werden lassen. Richard Wilhelms Arbeit gibt damit ein Musterbeispiel ab für die Rückwirkung kolonialer Politik in eine Situation, in der die kolonisierende Kultur in ihren expansiven Prämissen unsicher geworden und bereit ist, sich ihrerseits dem Fremden und Anderen der kolonisierten Kultur zuzuwenden. Wilhelm übersetzt und kommentiert in Tsingtau für den Verlag Eugen Diederichs unter anderem das alte Weisheits- und Orakelbuch „I Ging Das Buch der Wandlungen“, die Gespräche des Konfuzius („Lun Yü“) und die kanonischen Werke des Taoismus, „Dschuang Dsi - Südliches Blütenland“, „Liä Dsi - Quellender Ursprung“ sowie, mit der größten Breitenwirkung, 1911 das „Taoteking“ des Laotse, unter dem Titel „Das Buch des Alten vom Sinn und Leben“.

Die Zeitschriftenbeiträge belegen einen Wandel der Rezeptionshaltung, der die in ihrer philosophischen und literarischen Reichweite eminent folgenreiche Lektüre von Richard Wilhelms Übersetzungen und Kommentaren in Deutschland nachhaltig begünstigt haben dürfte. Was der Rezensent Friedrich Düsel noch etwas vage als "chinesische Religionsbücher“ angeführt hat, das legt Richard Wilhelm wenig später in kommentierten deutschen Übersetzungen Band für Band vor; Werktitel, die den Zeitschriftenautoren um 1900 noch sichtlich exotisch fremd und sperrig erschienen, werden von ihm in eine programmatisch dem Christentum und der Lutherbibel kompatible deutsche Sprache übertragen, inkulturiert. Wo um 1900 noch weithin, bei Düsel nicht anders als bei Grube, ein als überlegen voraus- 
gesetztes deutsches Wesen in ein als rückständig geltendes China exportiert werden sollte, da hat sich mit dieser Rezeption der kolonialpolitisch motivierte kulturwissenschaftliche Nachholbedarf nun vollends umgekehrt in eine Öffnung des deutschen Kulturlebens für Kunst, Literatur und Philosophie Chinas.

So beginnen nur wenige Jahre nach unserem Untersuchungszeitraum jene „chinesischen“ Dichtungen zu entstehen, die in der deutschsprachigen Frühmoderne eine so überraschend große Rolle spielen. Der expressionistische Romancier Alfred Döblin unternimmt schon 1912/13 mit seinem dem Liä-Dsi gewidmeten Chinaroman „Die drei Sprünge des Wang-lun“ eine emphatische Aufwertung der chinesischen Kultur; der junge Brecht liest 1920 Richard Wilhelms Übersetzung des „Taoteking“ ebenso begeistert wie der junge Hermann Hesse oder der Lyriker Klabund, dessen 1915 in der „Insel“-Bücherei erschienene Anthologie „Dumpfe Trommel und berauschtes Gong" die von Friedrich Düsel gerühmte Lyriksammlung Alfred Forkels in expressionistischer Emphase überbietet und rasch weiteste Verbreitung findet ${ }^{5}$. Der kulturelle Mentalitätswandel, der sich in den deutschen Kulturzeitschriften um 1900 in der Auseinandersetzung mit China abzeichnet, von Düsels Versuch einer kolonialen Instrumentalisierung sinologischen Wissens in „Westermanns Monatsheften“ bis zu den kolonialismuskritischen Vorstößen in Hardens „Zukunft“, markiert, so scheint es im Rückblick, den Beginn einer kulturellen China-Rezeption, die in ihrer Intensität, Offenheit und literarischen Produktivität in Deutschland bis dahin ohne Beispiel gewesen ist ${ }^{6}$.

\section{Anhang \\ Zeitschriftenbeiträge zur Wahrnehmung Chinas in Deutschland}

\section{Deutsche Rundschau}

98 (1899) 178-190 - M[ax] von Brandt: Tzse-Hsi. Kaiserin-Regentin von China und der Staatsstreich in Peking.

103 (1900) 96-112 - Wilhelm Grube: Der Confucianismus und das Chinesenthum. 104 (1900) 384-387 - M[ax] von Brandt: Die chinesische Frage.

$5 \quad$ Zu Entwicklung und Grundlinien dieser literarischen Rezeption bei Wilhelm, Hesse, Döblin, Klabund und Brecht vgl. im einzelnen (mit weiterführenden Literaturhinweisen) Detering.

6 In der Diskussion des vorliegenden Bandes in der Plenarsitzung der Akademie wies mich der Präsident darauf hin, daß sich ganz ähnliche Entwicklungen auch in der Rechtsdiskussion vollzogen haben. Vgl. zu diesem wichtigen, hier nicht weiter zu vertiefenden Bereich Heuser sowie grundlegend Starck. 
Westermanns illustrierte deutsche Monatshefte

85 (1899) 502-519 - M[ax] von Brandt: Ein Kapitel aus der chinesischen Kunstgeschichte. Symbolik und Bilderschrift.

85 (1899) 542 - F.D. [= Friedrich Düsel]: [Besprechung von Ernst von Hesse-Wartegg, Schantung und Deutsch-China in der Rubrik Litterarisches]

87 (1899) 140 f. - F.D. [= Friedrich Düsel]: [Besprechungen von Ernst Ruhstrat, Aus dem Lande der Mitte; Max von Brandt, Die chinesische Philosophie und der Staatsconfucianismus; Alfred Forke (Hg.), Blüten chinesischer Dichtung in der Rubrik Litterarische Rundschau].

87 (1899) 439 - F.D. [= Friedrich Düsel]: [Besprechung von Arthur H. Smith, Chinesische Charakterzüge].

89 (1900) 143 - F.D. [= Friedrich Düsel]: [Besprechung von Alfred Gründwedel, Mythologie des Buddhismus in der Rubrik Litterarische Rundschau].

89 (1900) 451 - F.D. [= Friedrich Düsel]: [Besprechung von Ferdinand Heigl, Religion und Kultur Chinas in der Rubrik Litterarische Rundschau].

89 (1901) 590 - F.D. [= Friedrich Düsel]: [Besprechung von Paul Goldmann, Ein Sommer in China. Zweite, vermehrte und verbesserte Auflage in der Rubrik Litterarische Rundschau].

89 (1901) 631-650 - M[ax] von Brandt: Peking sonst und jetzt und die frühen Hauptstädte des chinesischen Reiches.

89 (1901) 731 f. - F.D. [= Friedrich Düsel]: [Besprechungen von Ernst Schott, Die Wirren in China und ihre Ursachen; „Asiaticus“, Die Kämpfe in China; Dr. C. Spielmann, Die Taiping-Revolution in China; Eugen Wolf, Meine Wanderungen in der Rubrik Litterarische Rundschau unter der Sammelbezeichnung Die jüngste Litteratur über China].

Die Zukunft

30 (1900) 321-325 - [Maximilian Harden]: Tse-Si.

32 (1900) 49-55 - [Maximilian Harden]: Tsin-Schi-Hoang-Ti.

32 (1900) 97-103 - [Maximilian Harden]: Chinesisch-Deutsche Jahreszeiten.

32 (1900) 217-224 - [Maximilian Harden]: Kaiser von China.

\section{Literatur}

Debon, Günter: Goethes Chinesisch-Deutsche Jahres- und Tageszeiten in sinologischer Sicht, in: Euphorion 76 (1982), 27-57.

Detering, Heinrich: Bertolt Brecht und Laotse, Göttingen (Wallstein) 2008.

Döblin, Alfred: Die drei Sprünge des Wang-lun. Chinesischer Roman (Werke in Einzelbänden), München (dtv) 1989.

Forke, Alfred: Blüthen chinesischer Dichtung, Magdeburg (Faber) 1899.

Goethe, Johann Wolfgang: Chinesisch-deutsche Jahres- und Tageszeiten, in: ders: Sämtliche Werke. Gedichte 1800-1832. I. Abteilung: Band 2, hg. von Karl Eibl, Frankfurt/ M. (Deutscher Klassiker Verlag) 1988, 695-699.

Heuser, Robert: Beginn eines Jahrhundertprojekts: Die Rechtsreform unter der späten Qing-Dynastie (1903-1911), in: Zeitschrift für Chinesisches Recht 3 (2008), 193-205.

Klabund: Werke in acht Bänden, Bd. 7: Übersetzungen und Nachdichtungen, hg. von Christian von Zimmermann, Heidelberg (Elfenbein-Verlag) 2001. 
Kung Futse: Gespräche. Aus dem Chinesischen übertragen und erläutert von Richard Wilhelm, Jena (Diederichs) 1923.

Laotse: Taoteking. Das Buch des Alten vom Sinn und Leben. Aus dem Chinesischen übertragen und erläutert von Richard Wilhelm, Jena (Diederichs) 1911.

Struck, Wolfgang: Die Eroberung der Phantasie. Kolonialismus, Literaturund Film zwischen deutschem Kaiserreich und Weimarer Republik, Göttingen (Vandenhoeck \& Ruprecht) (Im Ersch.).

Starck, Christian: Gründe, Bedingungen und Formen von Rechtsrezeptionen, in: Heun, Werner/ Starck, Christian/ Tsai, Tzung-jen (Hgg.): Rezeption und Paradigmenwechsel im öffentlichen Recht. Viertes deutsch-taiwanesisches Kolloquium vom 7.-8 November in Taipeh (= Beiträge zum ausländischen und vergleichenden öffentlichen Recht 28), BadenBaden (Nomos) 2009, 25-35.

Tan, Yuan: Der Chinese in der deutschen Literatur. Unter besonderer Berücksichtigung chinesischer Figuren in den Werken von Schiller, Döblin und Brecht, Göttingen (Cuvillier) 2007.

Weber, Max: Die Wirtschaftsethik der Weltreligionen. Konfuzianismus und Taoismus. Schriften 1915-1920, hg. von Helwig Schmidt-Glinzer in Zusammenarbeit mit Petra Kolonko. Max-Weber-Studienausgabe, Bd. I/19, Tübingen (Mohr Siebeck) 1991. 